\title{
ZnO Thin Film Transistor: Effect of Traps and Grain Boundaries
}

\author{
A.A.Razak,'W.H.Khoo, S.M. Sultan* \\ Computational Nanoelectronics Research Group (CONE), Faculty of Electrical Engineering, Universiti Teknologi Malaysia, \\ 81310 UTM Johor Bahru, Johor, Malaysia
}

*Corresponding author: suhanasultan@utm.my

\begin{abstract}
Recently $\mathrm{ZnO}$ has drawn a lot of attention in semiconductor industry due to its interesting features. High exciton binding energy, high resistivity against radiation, high breakdown voltage, low temperature deposition are some of the interesting features of this material. Zinc oxide TFT device gains an increasing interest for its potential in sensing applications due to its biocompability, chemical stability and simple fabrication process with various methods and high surface-to-volume ratio. However, $\mathrm{ZnO}$ TFT devices from previous work exhibited poor $\mathrm{I}_{\mathrm{ON}}$ and field effect mobility. This work investigates the cause of its poor performance by focusing only two factors: traps and defects in the channel and grain boundary. The work was performed in Silvaco TCAD 2D simulator. It was found that a single grain boundary in the channel causes a reduction of the ION by $95 \%$. The effect in the IoN is less severe when traps and defects were introduced in the $\mathrm{ZnO}$ channel. The results can assist in optimizing the TFT device performance for sensing applications.
\end{abstract}

Keywords: TCAD simulation, TFT, $\mathrm{ZnO}$.

Article History: received 18 December 2016; accepted 7 February 2018; published 16 April 2018.

(c) 2018 Penerbit UTM Press. All rights reserved.

\section{INTRODUCTION}

Nowadays, thin film transistor (TFT) structure is gaining interest among academic and industry researchers not only for display applications but also as a sensing device. Various types of organic and inorganic materials were implemented in TFT structure as the active channel for chemical, biological, optical, magnetic, radioactive and other sensors by controlling the transport of charge carriers or emission of photon [1-4].Although TFT structure and its operation principles are the same as MOSFET but the fabrication involved is much simpler and hence more costeffective.

One of the semiconductor materials which is widely researched in TFT structure is zinc oxide, $\mathrm{ZnO}$. Due to its wide band gap at approximately $3.3 \mathrm{eV}$, it makes an interesting material for optical and sensor applications [5]. $\mathrm{ZnO}$ has the potential in the TFT technology for application in flexible and large-area electronics. Moreover, $\mathrm{ZnO}$ is attractive because of its relatively high charge carrier mobility and visual transparency [5]. These materials could build the foundation of thin film transistor technology for application in flexible and large-area electronics. Besides that, $\mathrm{ZnO}$ has excellent chemical and thermal stability and can be well oriented on various substrates [6].

Due to the ease of TFT structure and $\mathrm{ZnO}$ biocompatibility, $\mathrm{ZnO}$ TFT chemical sensor is an interesting device to be studied. However, $\mathrm{ZnO}$ film is known to be polycrystalline thin film [7]. The film has defects and grain boundaries which can impede the electron flow. Therefore, this work studies the effect of these defects and grain boundaries to the electrical characteristics of the $\mathrm{ZnO}$ TFT using basic device simulation. This is important in order to understand the key factors to further improve the TFT performance for chemical sensor applications.

\section{METHODOLOGY}

The ZnO TFT was designed by using SILVACO DevEdit tools virtually [8]. The important part of the design using DevEdit is meshing the structure. Meshing function is to get the precise and accurate points for characterization. Meshing in SILVACO allow the user control the detail of structure through multiple criteria; include material type, quality change, location or relative location [8].

The $\mathrm{ZnO}$ TFT structure is designed based on the work of S.M.Sultan et al [9]. The parameters of thin film transistor that were used are $10 \mu \mathrm{m}$ and $5 \mu \mathrm{m}$ for width and length respectively. The p-type substrate is highly doped which act as gate for this bottom gate structure. The doping concentration for the highly-doped bottom gate silicon is $1 \times 10^{20} \mathrm{~cm}^{-3}$ which degenerate the silicon gate into semiconductor level comparable to metals. The doping concentration of $\mathrm{ZnO}$ layer is set as $1 \times 10^{16} \mathrm{~cm}^{-3}$. Figure 1 shows the $\mathrm{ZnO}$ TFT structure.

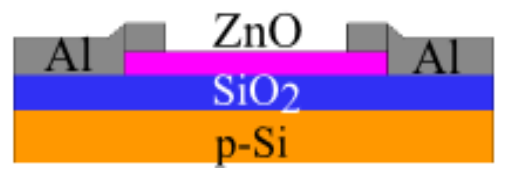

Figure 1. Schematic diagram of the TFT structure 


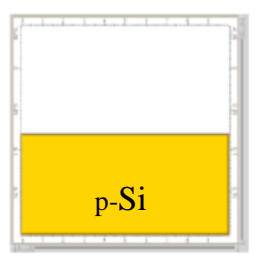

(a)

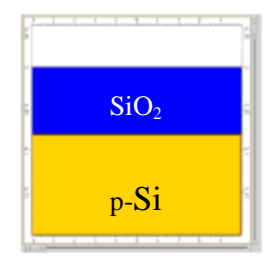

(b)

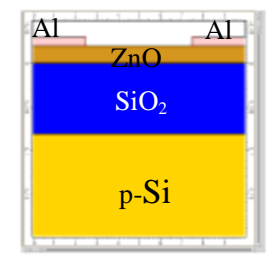

(d)

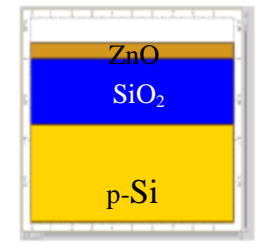

(c)
Figure 2. Fabrication steps virtually in two-dimensional

SILVACO TCAD Tools (a) highly doped silicon as bottom gate (b) silicon oxide (c) zinc oxide thin film and (d) Al electrodes as the source and drain

Figure 2 shows the step by step fabrication process of the TFT structure. The virtual fabrication step starts from the substrate of p-type silicon followed by the second layer which is $\mathrm{SiO}_{2}$ with $200 \mathrm{~nm}$ of thickness. The channel layer of this structure is zinc oxide which is connected to the source and drain layer with Aluminium material.

The analysis of the effect of defects and grain boundary was performed with the aid of numerical simulations using the Silvaco Atlas device simulator [8]. A 2D coupled Poisson's drift-diffusion model was used to investigate the TFT operation. In addition, model for carrier emission and absorption processes proposed by Shockley-Read-Hall (SRH) was used to reflect the recombination phenomenon within the device. The simulations were performed at room temperature.

The transistor parameters were extracted by using ATLAS commands. The $\mathrm{I}_{\mathrm{D}} / \mathrm{V}_{\mathrm{D}}$ and $\mathrm{I}_{\mathrm{D}} / \mathrm{V}_{\mathrm{G}}$ curves with different gate voltages were plotted in ATLAS. After obtaining the IV characteristics, the $\mathrm{ZnO}$ channel was introduced with various types of defects such as traps and a single grain boundary (GB) in the middle of the channel. Defects were introduced using the command defect in the simulation tool with trap densities of $7.5 \times 10^{18} \mathrm{~cm}^{-3}$. In all

\section{RESULTS \& DISCUSSION}

In the simulations, it was assumed that the interface of $\mathrm{SiO}_{2} / \mathrm{ZnO}$ layer is free of interface charges. The defects are only applied to the active layer of $\mathrm{ZnO}$ TFT. Figure 3 shows the semilogarithmic plot of the $\mathrm{I}_{\mathrm{D}}-\mathrm{V}_{\mathrm{G}}$ characteristics of the $\mathrm{ZnO}$ TFT with three different conditions; crystalline $\mathrm{ZnO}$ TFT, ZnO channel with traps in the channel and with a single grain boundary in the $\mathrm{ZnO}$ channel. Crystalline ZnO TFT shows a threshold voltage measured to be 1.36 $\mathrm{V}$. At $\mathrm{V}_{\mathrm{G}}=0 \mathrm{~V}$, the $\mathrm{I}_{\mathrm{D}}$ is $8 \mu \mathrm{A}$ and maximum current of $14.8 \mu \mathrm{A}$ is obtained at $\quad \mathrm{V}_{\mathrm{G}}=5 \mathrm{~V}$. This maximum current of $I_{D}$ at $V_{G}=5 \mathrm{~V}$ is termed as $I_{O N}$ in this paper.

When traps were introduced in the channel with trap densities of $7.5 \times 10^{18} \mathrm{~cm}^{-3}$, the threshold voltage was shifted positively to $2.83 \mathrm{~V}$. The $\mathrm{I}_{\mathrm{ON}}$ reduced to $5.5 \mu \mathrm{A}$ at $\mathrm{V}_{\mathrm{G}}=5 \mathrm{~V}$. Meanwhile when a single grain boundary was introduced in the $\mathrm{ZnO}$ channel, the threshold voltage shifted positively to $2.3 \mathrm{~V}$. However, I $\mathrm{I}_{\mathrm{ON}}$ reduced drastically to $0.17 \mu \mathrm{A}$.

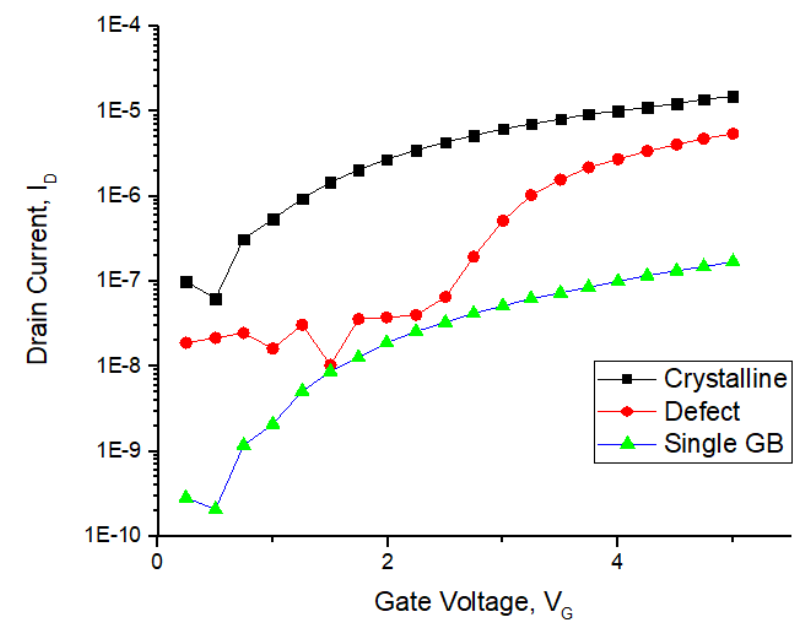

Figure 3. Semilogarithmic plot of the $I_{D}-V_{G}$ characteristics of the $\mathrm{ZnO}$ TFT with three different conditions

Figure 4 shows the contour plot of the electron flow in the $\mathrm{ZnO}$ TFT channel and its concentrations during these three conditions. For crystalline ZnO TFT, the depletion region under the drain electrode is more gradual at $V_{D}=1$ $\mathrm{V}$. However, when defects were introduced inside the $\mathrm{ZnO}$ channel, the depletion area under the drain region which is highlighted in yellow has increased. This indicates, more voltage is needed to ensure channel conductivity which results in higher threshold voltage as shown in the I-V characteristics earlier.

Figure 4(c) shows the contour plot when a single GB defined in the middle of the channel parallel to the $\mathrm{ZnO}$ film thickness and perpendicular to the direction of carrier propagation from source to drain. The GB is modeled as a thin layer (a few atomic layers) having defect states with Gaussian distribution as defined in Ref $[8,10]$. From the contour plot, depletion region starts to be visible after the GB region. It was reported in F.M.Hossein et al work that a double Schottky barrier is formed in the GB which impede the flow of electrons. As a result, when the threshold voltage is achieved, carriers transport over the GB will follow the thermionic emission process [7]. From the IV characteristics, the threshold voltage, $\mathrm{V}_{\mathrm{TH}}$ increases by $70 \%$ and the drain current, $I_{D}$ decreases by $95 \%$ when a single GB defined in the channel.

The increase of $\mathrm{V}_{\mathrm{TH}}$ and the decrease in $\mathrm{I}_{\mathrm{D}}$ affect the switching performance of the device. However, in P.Yasaei et al work, GB can enhance the sensitivity of the gas-sensing behaviour in graphene [11]. It was shown that the sensitivity of an isolated GB is x 300 times higher than that of a single graphene grain and much larger than that of polycrystalline graphene sensors [11].

Meanwhile when traps and defects were defined in the $\mathrm{ZnO}$ channel, the current decreases by only $63 \%$ from the crystalline $\mathrm{ZnO}$. $\mathrm{ZnO}$ thin film is well-known for its inherently high dope of n-type materials which always attributed to the oxygen vacancies [12]. These oxygen 
vacancies could be the possible source of traps. The oxygen vacancies in $\mathrm{ZnO}$ are in three states: $\mathrm{V}_{O}$ state which has captured two electrons and is neutral with respect to the lattice, the single ionized state $\mathrm{V}_{\mathrm{O}}{ }^{+}$and the $\mathrm{V}_{\mathrm{O}}{ }^{++}$which did not trap any electrons and is doubly positively charged. $\mathrm{V}_{\mathrm{O}}{ }^{+}$defect sites are found mostly at the surface of $\mathrm{ZnO}$ [13].These trap sites can cause the threshold voltage to shift positively because more electrons needed to be pumped into the active layer to overcome the trapped electrons. Consequently, higher electric field is needed in the channel layer.

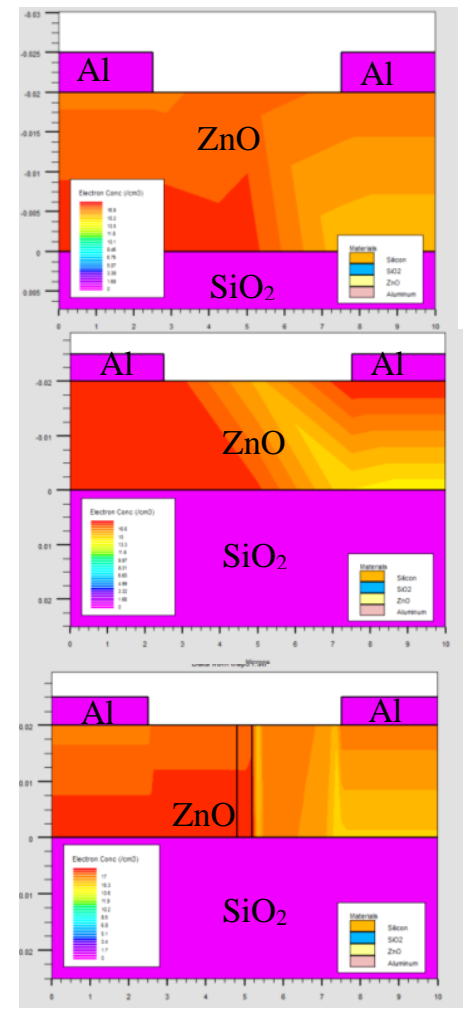

Figure 4 Contour plots of the electron concentration at different situations (a) crystalline ZnO TFT (b) when traps are introduced in the $\mathrm{ZnO}$ channel (c) when a single grain boundary introduced in the $\mathrm{ZnO}$ channel

\section{CONCLUSIONS}

Our TCAD simulations verify that the type of disorder (defect sites or grain boundary) is a determining factor in $\mathrm{ZnO}$ TFT performance. We have shown that a single GB in the active channel can have a large effect on the drain current. Meanwhile a high amount of defects in the $\mathrm{ZnO}$ channel cause a current decrease of $63 \%$ compared to $95 \%$ when a GB is defined in the channel. Therefore, it is important to determine the type of $\mathrm{ZnO}$ deposition process to be used in order to minimize grain boundaries in order to achieve optimized TFT performance for sensing applications.

\section{ACKNOWLEDGMENT}

This work was supported by Ministry of Education Malaysia through Fundamental Research Grant Scheme (FRGS) vot 4F482. The authors would like to acknowledge the support from UTM Research Management Centre.

\section{REFERENCES}

[1] P.Lin, F.Yan, "Organic thin-film transistors for chemical and biological sensing," Adv. Mater. vol. 24, pp. 34-51, 2012.

[2] J. Liu, M. Agarwal, K. Varahramyan, "Glucose sensor based on organic thin film transistor using glucose oxidase and conducting polymer,"Sensors and Actuators B:Chemical, vol. 135, pp. 195-199, 2008.

[3] E.Fortunato, P.Barquinha and R.Martins, "Oxide Semiconductor Thin-Film Transistors: A Review of Recent Advances,"Advanced Materials, vol.24, pp.2945-2986, 2012.

[4] Y. Kuo, "Thin film transistor technology--past present and future," Electrochemical Society Interface, vol.22, pp. 55-61, 2013.

[5] P. I. Reyes, C.-J. Ku, Z. Duan, Y. Lu, A. Solanki and $\mathrm{K}$.-B. Lee, "ZnO thin film transistor immunosensor with high sensitivity and selectivity," Applied Physics Letters, vol.98, pp. 173702, 2011.

[6] H.-C. Cheng, C.-F. Chen and C.-C. Lee, "Thin-film transistors with active layers of zinc oxide $(\mathrm{ZnO})$ fabricated by low-temperature chemical bath method," Thin Solid Films, vol.498, pp. 142-145, 2006.

[7] F. M. Hossain, J. Nishii, S. Takagi, A. Ohtomo, T. Fukumura, H. Fujioka, H. Ohno, H. Koinuma and M. Kawasaki, " Modeling and simulation of polycrystalline $\mathrm{ZnO}$ thin-film transistors," Journal of Applied Physics, vol. 94, pp. 7768-7777, 2003.

[8] Silvaco International, Atlas User's Manual Device Simulation Software, Silvaco International Ltd., Santa Clara, Dec., (2002).

[9] S. M. Sultan, O. D. Clark, T. Masaud, Q. Fang, R. Gunn, M. Hakim, K. Sun, P. Ashburn and H. M. Chong, " Remote plasma enhanced atomic layer deposition of $\mathrm{ZnO}$ for thin film electronic applications," Microelectronic Engineering, vol.97, pp. 162-165, 2012.

[10] M. Kimura, S. Inoue, T. Shimoda, and T. Sameshima, Jpn. J. Appl. Phys., Part 1, vol. 40, pp. 49, 2001.

[11] P.Yasaei et al, "Chemical sensing with switchable transport channels in graphene grain boundaries," Nature Communications, vol.5, Sept 2014.

[12] R.A. Street, " Thin-Film Transistors," Adv. Mater. vol.21, pp. 2007-2022, 2009.

[13] K.Vanheusden et al, "Mechanisms behind green photoluminescence in $\mathrm{ZnO}$ phosphor powders," Journal of Applied Physics, vol. 79, pp. 7983-7990, 1996. 